\title{
Estrutura de capital e custo de capital subsidiado no Brasil: influência no valor das ações das empresas
}

\author{
Capital structure and cost of subsidized capital in Brazil: influence in firm value
}

Estructura de capital y el costo de capital subvencionado en Brasil: implicaciones para el valor de las acciones de las empresas

\author{
Anderson Fioresi de Sousa \\ Mestre em Ciências Contábeis pela Fucape Escola de Negócios \\ Professor do Instituto Federal do Espírito Santo - IFES \\ CEP: 29645-000 - Santa Maria de Jetibá/ES - Brasil \\ E-mail: andersonfioresi@gmail.com \\ Telefone: (27) 2234-3000

\section{Fernando Caio Galdi} \\ Doutor em Ciências Contábeis pela USP \\ Professor da Fucape Escola de Negócios \\ Endereço: Av. Fernando Ferrari, n॰ 1358, Boa Vista \\ CEP: 29075-505 - Vitória/ES - Brasil \\ E-mail: fernando.galdi@fucape.br \\ Telefone: (27) 4009-4444
}

Endereço: Estrada Guilherme João Frederico Krüger, s/n, Caramuru

Artigo recebido em 28/05/2015. Revisado por pares em 14/07/2017. Reformulado em 02/10/2017. Recomendado para publicação em 08/03/2018 por Carlos Eduardo Facin Lavarda (Editor-Chefe). Publicado em 28/03/2018. 


\section{Resumo}

Grande parte do financiamento de longo prazo no Brasil é realizado por intermédio do Banco Nacional de Desenvolvimento Econômico e Social (BNDES). Comparado às outras alternativas do mercado este crédito é mais barato por ser subsidiado pelo governo. A presente pesquisa teve como intuito investigar se a captação de recursos com custo de capital subsidiado por meio de linhas de financiamento diferenciadas ofertadas pelo BNDES exerce influência no valor da empresa. $\mathrm{O}$ estudo foi desenvolvido com a utilização da metodologia estudo de eventos, que calcula os retornos anormais ocorridos ao redor de um determinado evento. A amostra deste estudo considera empresas listadas na Bolsa de Valores, Mercadorias e Futuros de São Paulo (BM\&FBOVESPA) que contrataram crédito junto ao BNDES de 2002 a 2015. Para o cálculo do retorno anormal foram usados dois modelos de estimação dos retornos anormais: o Modelo CAPM e o Modelo de Mercado. Os resultados indicam que a média dos retornos anormais das empresas que contrataram crédito junto ao BNDES é significativamente diferente de zero em torno da data do anúncio da contratação podendo-se concluir que a captação de recursos com custo subsidiado possui conteúdo informacional suficiente para influenciar o valor da empresa, confirmando a teoria do tradeoff das fontes de capital e a hipótese desta pesquisa.

Palavras-chave: BNDES; Custo subsidiado; Estrutura de capital; Mercado eficiente; Valor da empresa

\section{Abstract}

Much of the long-term funding in Brazil is conducted through the National Bank for Economic and Social Development (BNDES). Compared to other alternatives available in the market, this credit is cheaper, because it is subsidized by the federal government. This research aimed to investigate whether the fundraising with a capital cost subsidized by the government, through different funding lines offered by a government-controlled bank, has an influence on the value of the company. The study was developed by event study, which calculates abnormal returns occurring around a particular event. The sample considers companies that have used some sort of credit from BNDES from 2002 to 2015, and were listed on the Securities, Commodities and Futures Exchange Sao Paulo Stock Exchange (BM \& FBOVESPA). In order to calculate the abnormal returns, we used two models of estimation of returns: CAPM Model and Market Model. The results indicate that the average of the abnormal returns of the companies that contracted the loan with the BNDES is significantly different from zero around data of the announcement, being able to conclude that the fundraising with subsidized cost has the informational content sufficient to influence the value of the company, confirming the theory of the tradeoff of the sources of capital and the hypothesis of the research.

Keywords: BNDES; Capital structure; Efficient markets; Firm value; Subsidized cost

\section{Resumen}

Gran parte de la financiación de largo plazo en Brasil se lleva a cabo a través del Banco Nacional de Desarrollo Económico y Social (BNDES). En comparación con otras alternativas en el mercado, este crédito es más barato, ya que está subvencionado por el gobierno. El presente estudio se propone investigar si la recaudación de fondos con un costo subsidiado de capital a través de líneas de financiación diferenciado ofrecido por el BNDES, tiene influencia en el valor de la empresa. El estudio fue desarrollado con la utilización de la metodología del estudio de eventos, que calcula los retornos anormales ocurridos alrededor de un determinado evento. La muestra considera empresas que han contratado algún tipo de crédito de BNDES 2002 a 2015, y que fueron mencionados por los Valores, Mercaderías y Futuros de Sao Paulo Stock Exchange (BM \& FBOVESPA). Para el cálculo del retorno anormal se utilizaron dos modelos de estima de los retornos anormales: el Modelo CAPM y el modelo de mercado. Los 
resultados indican que el promedio de los retornos anormales de las empresas que contrataron el crédito junto al BNDES es significativamente diferente de cero en torno a datos del anuncio de la contratación, pudiéndose concluir que la captación de recursos con costo subsidiado tiene el contenido informacional suficiente para influenciar el valor de la empresa, confirmando la teoría del tradeoff de las fuentes de capital y la hipótesis de la investigación.

Palabras Clave: BNDES; Costo subsidiado; Estructura de capital; Mercados eficientes; Valor de la empresa

\section{Introdução}

Este estudo de evento investiga a reação do mercado ante a oferta de capital com custo subsidiado por linhas de crédito diferenciadas do Banco Nacional de Desenvolvimento Econômico e Social (BNDES), para financiamento às empresas. A hipótese assumida é de que a captação de recursos a um custo reduzido possua conteúdo informacional suficiente para mudar a expectativa dos investidores e gerar retornos anormais para as ações das empresas sujeitas ao evento.

O BNDES é um banco estatal com objetivo de democratizar o desenvolvimento econômico no Brasil. Os beneficiários dos seus financiamentos fazem uso de recursos públicos para financiamento de projetos e, caso tais operações possam ser usadas como ferramenta para geração de valor das empresas beneficiárias abre-se espaço para um universo de nuances políticas que poderiam extrapolar o papel de fomentador da economia praticado pelo banco (BACHILLER, 2016; TORRES FILHO; COSTA, 2011).

A participação do BNDES no financiamento das empresas cria um cenário de possível interferência na decisão de financiamento e, por conseguinte no valor da empresa, já que o custo de capital menor pressupõe aumento do valor desta (ALBANEZ et al., 2012; FAMÁ; GRAVA, 2000).

A teoria Trade-off, pela qual a busca por um custo de capital ótimo é ponto chave na formação de uma estrutura que reduza o custo de capital a um ponto otimizado é a base para supor que a ação do BNDES pode influenciar no valor das empresas (BASTOS; NAKAMURA, 2009; VALLE, 2008)

A partir do pressuposto de que financiamentos subsidiados, podem influenciar o valor da empresa financiada e, considerando que a previsibilidade deste fato ganha importância à medida que o investidor espera maximizar sua riqueza, torna-se relevante investigar como o mercado responde ao estímulo gerado por tais fatores. Assim, o objetivo desta pesquisa é responder a seguinte questão: o custo de capital subsidiado pelo governo gera reação anormal nos retornos das ações das empresas captadoras.

A contribuição da pesquisa está no fato de ajudar os investidores a entender se a política de financiamento a investimentos privados, com recursos públicos e custo subsidiado, promovida pelo governo pode afetar o mercado de capitais e, consequentemente, influenciar decisões dos stakeholders. Contribui ainda, abrindo precedentes para se vislumbrar desdobramentos políticos baseados em conflitos de interesse.

A seguir é apresentado um referencial teórico abordando as principais teorias sobre estrutura de capital, o cenário do crédito subsidiado, além da eficiência de mercado. A seção 3 traz a descrição do método estudo de evento. Nas seções 4 e 5 são apresentados os resultados e interpretações das estatísticas aplicadas. E na seção 6 as conclusões e recomendações para novos estudos. 


\section{Referencial Teórico}

\subsection{Estrutura de Capital e Valor da Empresa}

A maneira como as empresas financiam seus investimentos é tema de estudo há algum tempo. A teoria conhecida como tradeoff tem sido estudada há anos e sustenta a ideia de existência de uma estrutura de capital ótima. $\mathrm{O}$ aumento da dívida reduziria o custo de capital, contudo, fatores como o risco de falência tenderia a elevar tal custo, o que sugere a necessidade de um equilíbrio na formação do capital (CORREA; BASSO; NAKAMURA, 2013; HARRIS; RAVIV, 1991; MODIGLIANI; MILLER, 1958; SILVA BRITO; CORRAR; BATISTELLA, 2007)

Outras teorias com abordagens comportamentais e estruturais são bastante conhecidas: A teoria d agência na qual a estrutura de capital é usada para restringir decisões dos gerentes (HARRIS; RAVIV, 1991; JENSEN; MECKLING, 1976; VALLE, 2008); a teoria do pecking order que estabelece uma hierarquia com base na assimetria da informação, dando preferência a recursos gerados internamente frente a recursos externos e, caso decidisse por estes, a emissão de dívida antecederia a emissão de ações (CORREA; BASSO; NAKAMURA, 2013, 2013; FAMÁ; GRAVA, 2000; MYERS, 1984)

Trabalhos importantes como Titman (1984), Titman e Wessels (1988) Rajan e Zingales (1995), Myers (2001), Grinblatt e Titman (2005); Valle e Albanez (2012); Grzebieluckas et al. (2017); Tarantin Junior e Valle (2015); Kudlawicz, Senff e Bach (2015); Locatelli, Nasser e Mesquita (2015); Correa, Basso e Nakamura (2013) tem sido realizados com o intuito de compreender ao longo das últimas décadas quais fatores são determinantes da estrutura de capital, bem como suas consequências no custo deste capital.

Albanez et al., 2012; Valle e Albanez, 2012; Tarantin Junior e Valle, 2015; Valle, (2008) tem destacado o papel das fontes de financiamento subsidiadas na formação da estrutura de capital das empresas com destaque para as linhas de financiamento do BNDES. Os trabalhos, contudo, não fazem qualquer abordagem a geração de valor por parte das empresas financiadas, tal como no presente estudo.

Não obstante a tantas abordagens que circundam o tema, a base teórica para a presente pesquisa fundamenta-se na teoria do trade-off. Num cenário em que o custo de capital de terceiros é subsidiado pelo governo, espera-se que o benefício fiscal seja mais expressivo do que seria sem subsídio. Assim, o custo médio do capital deverá apresentar uma relação inversa ao subsídio, ou seja, quanto mais subsídio para o custo do capital de terceiros, menor será o CMPC e, consequentemente, maior o valor da empresa.

\subsection{Teoria da Eficiência de Mercado}

Segundo a teoria da eficiência de mercado, os preços de um ativo devem sempre refletir plenamente todas as informações disponíveis (FAMA, 1970). Pressupõe-se assim, que num mercado eficiente, informações relevantes não são capazes de mudar a expectativa dos investidores em relação a ganhos futuros e, consequentemente os preços das ações não sofrem impacto.

Fama (1970) atribui três classificações para um mercado eficiente: forma fraca, em que toda a informação relevante refletida no preço da ação está contida apenas em seus preços históricos; forma semiforte, em que os preços refletem plenamente, não apenas os preços históricos, mas também toda informação pública relevante; e forma forte, em que os preços refletem todas as informações, públicas ou privadas.

Camargos e Barbosa (2006) investigaram se o mercado brasileiro é eficiente na forma semiforte através de um estudo de eventos dos anúncios de fusões e aquisições de firmas 
listadas na Bolsa de Valores entre 1994 e 2002. Concluíram que o mercado não se comportou de maneira eficiente.

Barbosa e Medeiros (2007), Camargos e Barbosa (2015), Malaquias e Eid Junior, (2013), Gabriel, Ribeiro e Ribeiro, (2013), Gaio, Freitas Alves e Pimenta Júnior (2009) são outros estudos que também pesquisaram sobre a eficiência do mercado acionário brasileiro e concluíram que não há indícios de característica de mercado eficiente.

O que a teoria de eficiência de mercado sustenta na presente pesquisa é que num cenário com fatores institucionais, caracterizado por linhas diferenciadas de financiamento com custo abaixo do mercado, pode-se esperar que empresas se sintam atraídas a compor sua estrutura de capital com este tipo de fonte de recursos e que o mercado talvez reaja a este evento. Caso o mercado capte este evento, que representa diminuição do custo de capital pode-se intuir que a decisão de estrutura de capital motivada pelo custo subsidiado pelo governo possua conteúdo informacional suficiente para influenciar o valor de negociação das ações da empresa.

\subsection{Custo de Capital Subsidiado e o Papel do BNDES}

Bancos de desenvolvimento têm um papel importante, principalmente, em países subdesenvolvidos. Schapiro (2009) destaca que a participação do Estado assumindo a função de agente financeiro e sendo responsável pelo estímulo econômico é uma terceira via de acesso a recursos, além do mercado de capitais e dos bancos privados. E esta via ganha importância em países em desenvolvimento, os quais, normalmente, possuem mercado de capital pouco desenvolvido, além de apresentarem ambiente econômico com maior risco inibindo a atuação de bancos privados (BACHILLER, 2016; TARANTIN JUNIOR; VALLE, 2015; KRASNER, 1981; TORRES FILHO; COSTA, 2011).

O Brasil, como um país em desenvolvimento, insere-se neste contexto, tendo o Banco Nacional de Desenvolvimento Econômico e Social (BNDES) como agente estratégico em sua política de desenvolvimento. O país possui como característica marcante a participação do estado no financiamento ao investimento privado sendo que as principais fontes de recursos, geralmente, não vêm do setor privado, como é comum em outros mercados, mas sim de fontes fornecidas pelo próprio governo, principalmente através do BNDES (BACHILLER, 2016; PRATES; CINTRA; FREITAS, 2000; TORRES FILHO; COSTA, 2011).

Historicamente, o BNDES atua como articulador financeiro de políticas desenvolvimentistas dando suporte a projetos de investimento em infraestrutura e exercendo papel anticíclico de oferta de crédito em momentos de forte aversão ao risco, por parte de instituições privadas, como forma de estabilização da economia (BACHILLER, 2016; FERRAZ et al., 2012; PRATES; CINTRA; FREITAS, 2000).

Após atuar como agente executor de privatizações da década de 90 o BNDES voltou a atuar mais próximo da agenda de desenvolvimento durante os anos 2000 , sendo o principal financiador do programa de aceleração do crescimento (PAC) e apresentando relevante crescimento no volume recursos aplicados nos projetos de investimento de seus beneficiários, principalmente a partir de 2008 (BACHILLER, 2016; TARANTIN JUNIOR; VALLE, 2015).

Apesar de toda a relevância do BNDES como agente de desenvolvimento no Brasil, não foram encontrados estudos que relacionem a participação destes recursos na estrutura de capital das empresas com o valor destas. A hipótese assumida no presente estudo é de que o anúncio da contratação de financiamento do BNDES com custo subsidiado gera anormalidade positiva nos retornos das ações das empresas sujeitas ao evento. 


\section{Metodologia}

Para atingir o objetivo da pesquisa foi realizado um estudo de eventos, método que tenta examinar o comportamento dos retornos de um grupo de empresas que experimentaram um evento comum (KOTHARI; WARNER, 2005). Camargos e Barbosa (2003) enfatizam que os estudos de eventos são amplamente utilizados em finanças e trata-se de um método comum aos testes de eficiência de mercados na forma semiforte, ou seja, na reação dos preços a anúncios públicos.

\subsection{Estudo de Eventos}

Esta metodologia baseia-se num modelo de geração de retornos de ações. Com base neste modelo é determinado o retorno normal, que é o retorno esperado da ação caso não ocorra o evento. Em seguida, este retorno será comparado com os retornos observados durante um determinado período a fim de se verificar a existência de comportamento anormal dos retornos em períodos próximos ao evento. $\mathrm{O}$ aumento da variância dos retornos próximo à ocorrência do evento pressupõe que tal evento possui conteúdo informacional relevante (CARMARGOS; BARBOSA, 2003).

\subsection{Definição do evento}

A utilização do estudo de evento requer a definição de um evento de interesse, da data de sua ocorrência, denominada "data zero", e do período em que serão examinados os preços das ações, chamada de "janela de evento". No presente estudo o evento que se espera seja capaz de gerar comportamentos anormais nos retornos é a captação de recursos com custo subsidiado através de linhas de financiamento diferenciadas, representadas neste caso pelas linhas de crédito do BNDES. As linhas de crédito do BNDES têm custo diferenciado a fim de atrair e alavancar o desenvolvimento econômico do país (ALBANEZ et al., 2012; VALLE; ALBANEZ, 2012; TARANTIN JUNIOR; VALLE, 2015; VALLE, 2008).

Existem alguns momentos passíveis de serem considerados como a data de ocorrência do evento para verificação do objetivo desta pesquisa, sendo talvez o principal deles, a data da decisão pelo financiamento, junto ao BNDES, manifestada através do anúncio de um novo projeto de investimento.

Contudo, a possível utilização do momento da decisão de financiamento - data em que a intenção de a empresa tomar crédito junto ao BNDES chega ao mercado, provavelmente pela divulgação de um novo projeto - como data zero não foi considerada neste estudo devido influência de outros eventos. Como é sabido, as decisões de investimento e de financiamento são tomadas sempre em conjunto e, caso o mercado responda a esta informação, é improvável que se consiga dissociar qual aspecto, se a decisão de financiamento ou de investimento, possui conteúdo informacional e em qual proporção. Esta dificuldade é esperada quando se trata de pesquisas sobre estrutura de capital, podendo-se considerar praticamente impossível separar as decisões de investimento e financiamento (COPELAND; WESTON; SHASTRI, 1983)

Procianoy e Antunes, (2001), que investigaram exatamente o impacto dos investimentos no valor das ações, destacam que a maioria das pesquisas sobre o tema tem por base a data do anúncio. Contudo, o momento da decisão de investimento, por parte da empresa, reflete apenas a existência de uma decisão de aplicar recursos em um projeto que, contudo, pode não ter sua fonte de recursos definida e, apesar de existir a expectativa em relação ao financiamento, a captação pode ser feita junto a outras fontes, o que não configuraria em custo subsidiado, como propõe o presente estudo. 
O momento em que a redução do custo de capital se estabelece é na contratação do crédito junto ao BNDES, de tal modo que a data de anúncio desta contratação é o ponto que melhor representa a data de evento, uma vez que o mercado tenderá a reagir assim que tomar conhecimento desta informação (BARBOSA; MEDEIROS, 2007; MALAQUIAS; EID JUNIOR, 2013; GAIO; FREITAS ALVES; PIMENTA JÚNIOR, 2009). Assim, nesta pesquisa a data do anúncio da contratação do financiamento, por meio de fatos relevantes e/ou comunicados ao mercado, é a data do evento, na qual será testada a existência de retornos anormais.

Para mensuração do retorno anormal foram determinadas três janelas de evento, com períodos antes e depois da data do evento: a primeira considerando o período de um dia antes e um dia depois da data zero $(-1 \mathrm{a}+1)$, chamada de janela $1(\mathrm{~J} 1)$; a segunda considerando três dias antes e três dias depois da data zero $(-3 \mathrm{a}+3)$, chamada de janela 2 (J2); e a terceira considerando sete dias antes e sete dias depois da data zero $(-7 \mathrm{a}+7)$, chamada de janela $3(\mathrm{~J} 3)$. O período de estimação dos retornos normais foram os 180 dias anteriores à janela de eventos, (de -187 a -8). A definição do tamanho das janelas de evento e estimação estão em linha com Murcia, Murcia e Borba (2013); Gabriel, Ribeiro e Ribeiro (2013); Procianoy e Antunes (2001).

As janelas de evento e de estimação são definidas a critério do pesquisado podendo se estender por vários dias e, inclusive, compreender o momento antes do evento, visto que o mercado pode ter recebido a informação antes do real acontecimento do evento (MACKINLAY, 1997; CAMARGOS; BARBOSA, 2003). A figura 1 apresenta um esquema com as janelas de evento.

Figura 1: Janelas de Evento

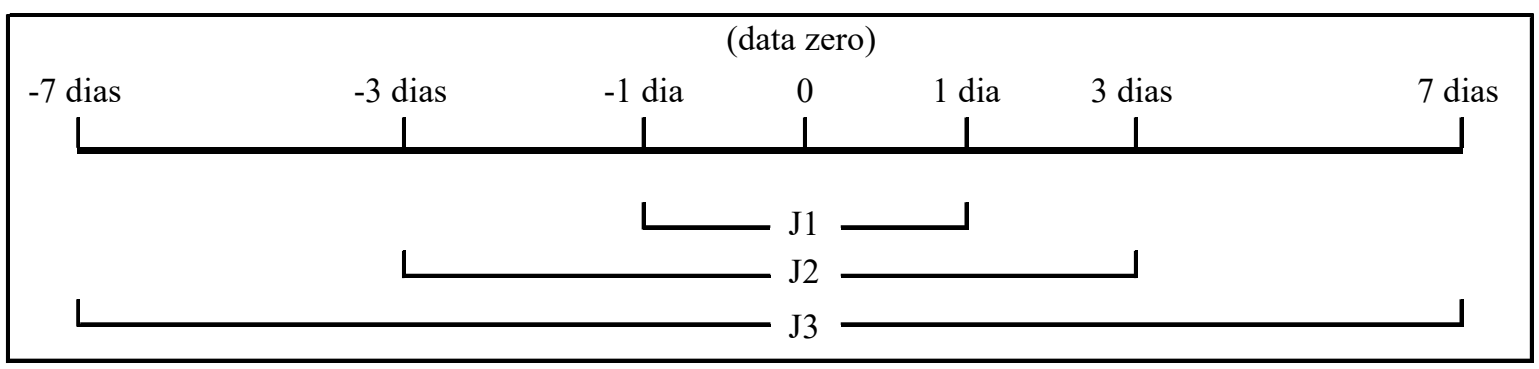

Fonte: Elaborada pelos autores.

\subsection{Critérios de Seleção}

A amostra foi selecionada a partir de consulta on-line ao banco de informações da Comissão de Valores Mobiliários - CVM. Os Fatos Relevantes e os Comunicados ao Mercado emitidos pelas companhias e que fazem referência ao BNDES em seus títulos foram consultados e selecionados apenas os que se tratavam de anúncio de contratação de financiamento junto ao banco.

Apesar da existência de operações de crédito com o BNDES que não foram anunciados ao mercado por meio de um dos tipos de documentos citados, a escolha exclusiva destes documentos nesta pesquisa é a que melhor representa o anúncio da contração de financiamento minimizando o risco de sobreposição de eventos de outra natureza.

Foram encontradas 136 companhias que emitiram Fatos Relevantes ou Comunicados ao Mercado que fazem referência ao BNDES no período de 2002 a 2015. Após análise dos documentos e exclusão de companhias com ações ilíquidas na janela de eventos, a amostra foi composta com 40 empresas que emitiram 62 documentos com anúncio de contratação de crédito 
junto ao BNDES, ou seja, em alguns casos a mesma empresa contratou mais de uma operação de crédito.

A tabela 1 mostra as ações representativas das 40 empresas selecionadas para a amostra, bem como a quantidade de contratações de financiamento junto ao BNDES anunciadas através de fatos relevantes ou comunicados ao mercado no período da pesquisa.

Tabela 1 - Ações e quantidades de anúncios

\begin{tabular}{|c|c|c|c|}
\hline Ação & $\mathrm{N}^{0}$ anúncios & Ação & $\mathrm{N}^{0}$ anúncios \\
\hline ALPA3 & 1 & IGTA3 & 2 \\
\hline ARTR3 & 6 & JHSF3 & 1 \\
\hline BEMA3 & 1 & LINX3 & 2 \\
\hline CLSC4 & 2 & LOGN3 & 3 \\
\hline FESA3 & 1 & LAME4 & 1 \\
\hline CGAS3 & 4 & LUPA3 & 1 \\
\hline PRVI3 & 1 & BEEF3 & 1 \\
\hline CSMG3 & 1 & OIBR4 & 1 \\
\hline SAPR4 & 1 & OSXB3 & 1 \\
\hline CNFB4 & 1 & PDGR3 & 1 \\
\hline CTAX4 & 3 & PETR3 & 1 \\
\hline ECOR3 & 1 & PRML3 & 2 \\
\hline ENBR3 & 3 & SMTO3 & 2 \\
\hline ELPL5 & 1 & SLED4 & 1 \\
\hline EMAE4 & 1 & SNSL3 & 1 \\
\hline ENEV3 & 3 & SUZB5 & 2 \\
\hline EGIE3 & 1 & TPIS3 & 1 \\
\hline FHER3 & 1 & FFTL4 & 1 \\
\hline GOLL4 & 1 & VALE3 & 1 \\
\hline GVTT3 & 1 & VLID3 & 1 \\
\hline
\end{tabular}

Fonte: Elaborada pelos autores

O valor dos financiamentos totaliza $\mathrm{R} \$ 77,8$ bilhões e representa uma relação média Financiamento/Ativo de 12,6\%. A relação média Dívida Bruta/Ativo das empresas da amostra é de $47 \%$, de tal modo que a média dos financiamentos obtidos representa $26,8 \%$ da média da dívida bruta das empresas.

Em relação à distribuição temporal dos eventos, observa-se que a partir de 2009 houve forte crescimento na quantidade de financiamentos realizados pelo BNDES. Este período caracterizou-se como um momento de desenvolvimento no mercado de capitais brasileiro além de expansão na oferta de crédito por instituições públicas (TARANTIN JUNIOR; VALLE, 2015).

A tabela 2 mostra a quantidade de contratações de financiamentos junto ao BNDES por ano no período da pesquisa.

Tabela 2 - Quantidade de anúncios por ano

\begin{tabular}{c|c|c|c}
\hline Ano do anúncio & Quantidade de anúncios & Ano do anúncio & Quantidade de anúncios \\
\hline 2002 & 1 & 2009 & 14 \\
\hline 2003 & 0 & 2010 & 11 \\
\hline 2004 & 1 & 2011 & 6 \\
\hline 2005 & 4 & 2012 & 8 \\
\hline 2006 & 2 & 2013 & 6 \\
\hline 2007 & 3 & 2014 & 2 \\
\hline 2008 & 4 & 2015 & \\
\hline
\end{tabular}

Fonte: Elaborada pelos autores 
Ilustrativamente, o gráfico 1 mostra a linha temporal dessas contratações distribuídas por ano no período da pesquisa.

Gráfico 1 - Distribuição dos anúncios por ano

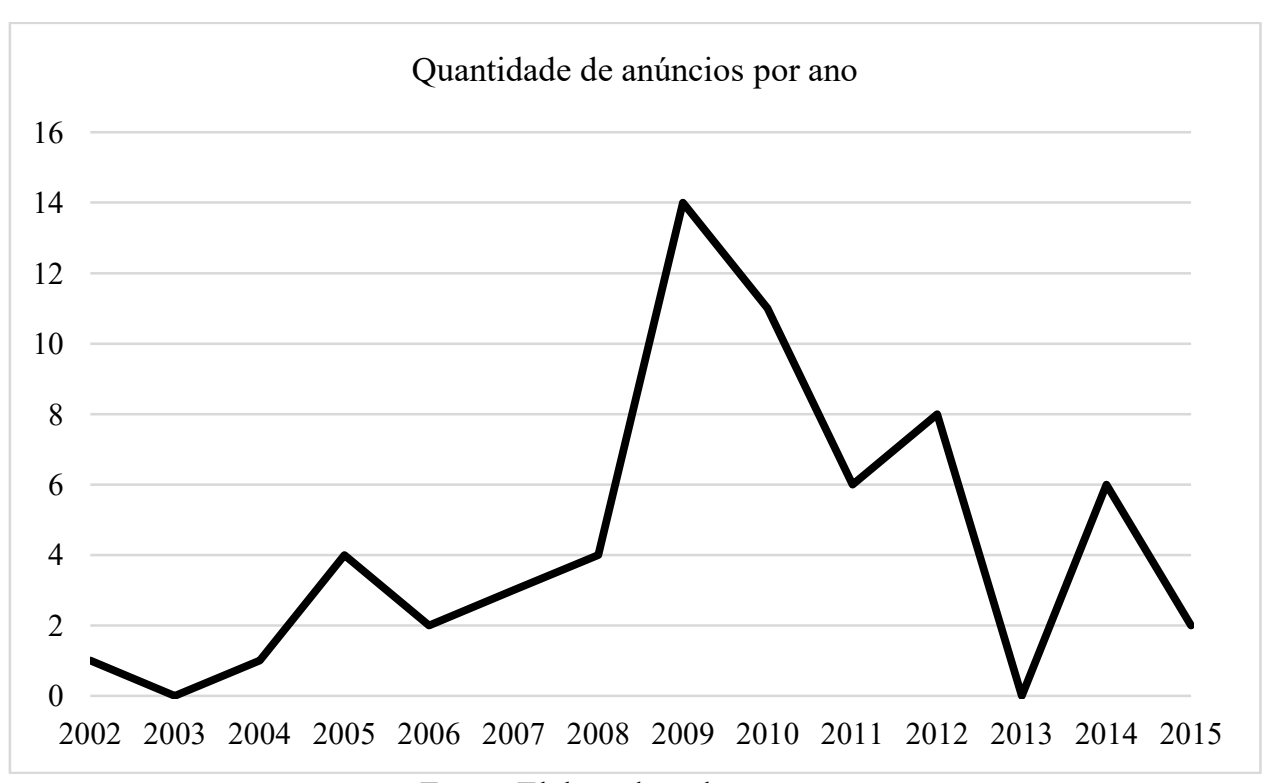

Fonte: Elaborado pelos autores

\subsection{Retornos Normais e Anormais}

O retorno normal é obtido através da divisão entre as cotações diárias do período atual e período anterior sendo esta aplicada em sua forma logarítmica que tende a aproximar a curva de distribuição de frequências dos retornos à curva de distribuição normal (CAMARGOS; BARBOSA, 2015; KOTHARI; WARNER, 2005; SALINGER, 1992; CAMARGOS; BARBOSA, 2003). Assim, o retorno normal é dado por:

$$
R_{i t}=\ln \left(P_{i t} / P_{i t-1}\right)(1)
$$

sendo, $\mathrm{R}_{\mathrm{it}}, \mathrm{P}_{\mathrm{it}} \mathrm{e} \mathrm{P}_{\mathrm{it}-1} \mathrm{o}$ retorno da ação $i$ na data $t$, o preço da ação $i$ na data $t$, e o preço da ação i na data $t-1$, respectivamente.

Algumas empresas, com menor liquidez, apresentaram dias sem negociação durante as janelas de estimação e de evento, assim, foi utilizado o procedimento Uniform Return Procedure, que distribui o retorno, da próxima data com negociação, de maneira uniforme entre as datas sem negociação (BARTHOLDY OLSON;; PEARE, 2007).

O retorno normal esperado, por sua vez, deve ser estimado com base em algum modelo de previsão de retornos. Brown e Warner (1985) sugerem três modelos que são geralmente usados em estudos de eventos: modelo de retornos médios ajustados; modelo de retornos ajustados ao mercado; e Capital Asset Price Modelo (CAPM), sendo que os dois últimos foram utilizados no presente estudo.

O modelo CAPM, foi o primeiro a ser escolhido por que além de atender aos pressupostos estatísticos, obedece a restrições econômicas, o que tende a melhorar a precisão dos retornos normais (CAMARGOS; BARBOSA, 2003). A equação 2 apresenta o cálculo do retorno esperado:

$$
E\left(R_{i t}\right)=R_{r f}+\beta_{i}\left[E\left(R_{m t}\right)-R_{r f}\right](2)
$$


Sendo, $\mathrm{E}\left(\mathrm{R}_{i t}\right), \mathrm{R}_{\mathrm{rf}}, \mathrm{R}_{m t}$ e $\beta_{i}$, retorno observado do título $i$ no período $t$, retorno livre de risco, retorno observado da carteira de mercado no período $t$ e risco sistemático da ação diante do mercado, respectivamente.

O retorno normal observado foi obtido através da equação (1) aplicada nas janelas de evento, sendo os dados relativos aos preços dos títulos coletados do banco de dados Economática. Na equação (2), a taxa livre de risco adotada foi a taxa CDI diária obtida do site da CETIP, geralmente aceita no mercado Brasileiro para este fim. O retorno de mercado utilizado foi o IBRX índice Brasil e o $\beta$ estimado com base nos 60 meses anteriores a data de evento, ambos obtidos no banco de dados da Economática.

Os retornos anormais são obtidos pela diferença entre os retornos observados do título $i$ no tempo $t$, na janela de eventos, e os retornos esperados, estimados de acordo com o modelo de estimação dos retornos normais, demonstrado na equação 2 . Assim, tem-se que:

$$
R A_{i t}=R_{i t}-E\left(R_{i t}\right)(3)
$$

sendo, RAit, Rit e E(Rit), retorno anormal do título i no período t, retorno observado do título i no período t e retorno esperado do título i no período $t$, respectivamente.

O segundo modelo de estimação dos retornos foi o modelo de retornos ajustados ao mercado, também conhecido como modelo de mercado. $\mathrm{O}$ objetivo é proporcionar maior robustez aos resultados, uma vez que o modelo anterior é sensível aos efeitos da estimação do beta para casos de ações com pouca liquidez. Procedimento semelhando foi adotado por Murcia, Murcia e Borba (2013). Neste modelo os retornos anormais são calculados conforme definido na equação 4:

$$
R A_{i t}=R_{i t}-R_{m t}(4)
$$

Sendo, $R_{m t}$ o retorno observado da carteira de mercado no período t.

\subsection{Procedimentos de Teste}

O teste estatístico que geralmente é usado para a determinação da significância dos retornos anormais consiste em testar a hipótese nula de que a média dos retornos anormais é igual a zero (KOTHARI; WARNER, 2005). Para isso, utilizou-se técnica de acumulação dos retornos sugerida em Kothari e Warner (2005), de tal modo que os retornos anormais diários foram acumulados (RAA) através da simples soma no período da janela de evento, conforme equação 5 .

$$
R A A_{i}\left(t_{1}, t_{2}\right)=\sum_{i=i t}^{t 2} R A_{i t}(5)
$$

Sendo $t_{1} e t_{2}$, primeiro e último dia de cada janela de evento.

Os dados são analisados em cross-section, uma vez que, a partir da acumulação dos retornos anormais para cada título, os mesmos foram analisados num determinado momento e submetidos a testes estatísticos realizados através do software STATA.

Caso o retorno anormal acumulado médio (CAR) seja estatisticamente diferente de zero, tem-se que os retornos obtidos na janela de evento desviaram do retorno esperado confirmando a hipótese da pesquisa de que o evento possui conteúdo informacional. O CAR é obtido através da equação 6 .

$$
C A R=\frac{1}{N} \sum_{i=1}^{n} R A A_{i}(6)
$$




\section{Resultados}

\subsection{Teste de Significância dos Retornos Anormais}

O primeiro passo foi calcular o retorno anormal diário de cada empresa e em cada janela de estimação e na janela de evento, conforme descrito nas equações 1, 2 e 3. Em seguida, os retornos anormais diários foram acumulados nas respectivas janelas para cada evento, conforme equação 5 .

Enfim, a média dos retornos anormais acumulados (CAR), calculado conforme equação 6 , é testado sob a hipótese de ser significativamente diferente de zero.

Para cada uma das três janelas de evento foi realizado o teste de normalidade de ShapiroWilk, a fim de se verificar se a distribuição transversal dos retornos anormais dos títulos se aproxima da distribuição normal, que é condição para realização do teste paramétrico.

Após o teste de normalidade, os retornos anormais acumulados dos títulos nas três janelas não se assemelham à distribuição normal, sendo mais indicada a utilização de teste não paramétrico para avaliar o retorno anormal (SALINGER, 1992). Assim, foi aplicado o teste de sinais, não paramétrico Wilcoxon signed rank test, conforme adotado em Brown e Warner (1980).

A Tabela 3 mostra o teste não paramétrico de Wilcoxon. Nela estão discriminados o teste não paramétrico e o respectivo valor-p para todas as janelas.

Tabela 3 - Teste não Paramétrico Wilcoxon Signrank

\begin{tabular}{c|c|c}
\hline & $\mathrm{z}$ & $\operatorname{Pr}>|\mathrm{z}|$ \\
\hline $\mathrm{J} 1$ & 1,125 & 0,2605 \\
\hline $\mathrm{J} 2$ & 1,833 & $0,0667 *$ \\
\hline $\mathrm{J} 3$ & 0,046 & 0,9637 \\
\hline \multicolumn{2}{r|}{$* * *, * * *$, nível de significância de 10\%, 5\% e 1\% respectivamente } \\
\hline
\end{tabular}

Fonte: Elaborada pelos autores.

Observa-se que na janela J2 a hipótese nula foi rejeitada a um nível de significância de $10 \%$. Pode-se afirmar que o retorno anormal acumulado dos títulos no período de três dias antes e depois do evento é diferente de zero, implicando que a contratação de crédito junto ao BNDES impactou positivamente o valor das ações confirmando a hipótese desta pesquisa.

A fim de conferir maior robustez ao estudo, foi procedido um segundo teste considerando, porém, os retornos anormais calculados com base no modelo de retornos ajustados ao mercado, conforme descrito na equação 4. A tabela 4 mostra o teste não paramétrico de Wilcoxon para os retornos anormais calculados neste modelo. Nela estão discriminados o teste não paramétrico e o respectivo valor-p para todas as janelas.

Tabela 4 - Teste não Paramétrico Wilcoxon Signrank

\begin{tabular}{c|c|c}
\hline & $\mathrm{Z}$ & $\operatorname{Pr}>|\mathrm{z}|$ \\
\hline $\mathrm{J} 1$ & 1,623 & 0,1046 \\
\hline $\mathrm{J} 2$ & 1,882 & $0,0598^{*}$ \\
\hline $\mathrm{J} 3$ & 0,214 & 0,8307 \\
\hline \multicolumn{2}{r|}{$, * *, * * *$, nível de significância de $10 \%, 5 \%$ e $1 \%$ respectivamente } \\
\hline
\end{tabular}

Fonte: Elaborada pelos autores.

O resulta deste teste confirma o resultado encontrado para os retornos estimados com base no modelo CAPM. Na janela J2 a hipótese nula foi rejeitada a um nível de significância de 
$10 \%$, de tal modo que o retorno anormal acumulado dos títulos no período de três dias em torno da data do evento é diferente de zero.

Estes resultados estão em linha com o que preconiza a teoria tradeoff, ou seja, o estabelecimento de uma estrutura de capital que otimiza o custo de capital promove aumento no valor da empresa (CORREA; BASSO; NAKAMURA, 2013; MODIGLIANI; MILLER, 1958; MYERS, 1984).

O resultado também encontra aderência em outros trabalhos recentes tais como Valle e Albanez (2012); Tarantin Junior e Valle (2015); Valle (2008) que relacionam subsídios governamentais com a estrutura de capital das empresas sem, porém, abordar o aspecto valor da empresa.

Ademais, os achados de Peixoto et al. (2016) corroboram os aqui encontrados. A pesquisa indica que há indícios de relação negativa entre práticas sustentáveis medida pelo Índice de Sustentabilidade Empresarial e que contribui para melhor gestão de risco interno e o custo de capital.

\section{Considerações Finais}

A pesquisa teve por objetivo investigar a reação do mercado ante a oferta de capital com custo subsidiado por linhas de crédito diferenciadas do Banco Nacional de Desenvolvimento Econômico e Social (BNDES), para financiamento das empresas. A hipótese de pesquisa foi de que a captação de capital a um custo reduzido possuísse conteúdo informacional suficiente para mudar a expectativa dos investidores e gerar retornos anormais para as ações das empresas.

O estudo foi desenvolvido com a metodologia o estudo de eventos, que calcula os retornos anormais ocorridos ao redor de um determinado evento, que neste caso foi a contratação de financiamento junto ao BNDES.

Os resultados confirmaram a hipótese de pesquisa, de tal modo que os testes estatísticos indicaram que a média dos retornos anormais obtidos pelas empresas que contrataram crédito junto ao BNDES foi positiva e significativa, num período de três dias em torno da data da contratação.

A limitação do estudo reside na dificuldade de se determinar o exato momento de ocorrência do evento, ou seja, o anúncio da contratação de crédito. Empresas fazem contratação do mesmo tipo de crédito sem, entretanto, comunicar o mercado em documento específico. Esta discussão abre uma lacuna para outros estudos repetirem a investigação considerando outros tipos de evidenciação da informação ao mercado.

A principal porta que a presente pesquisa abre para investigações futuras, entretanto, assenta-se sobre o viés político e de conflito de interesses que o resultado sugere. Quais critérios e condutas devem ser seguidas na concessão deste tipo de financiamento, visto que os benefícios oriundos daí extrapolam o aspecto desenvolvimentista e adentram no âmbito do mercado financeiro?

O presente estudo tomou como base apenas os créditos ofertados pelo BNDES, entretanto, em linha com Valle e Albanez (2012), existem outras opções de linhas diferenciadas que podem ser entendidas como subsídio para o custo de capital, tais como recursos oriundos de outros Bancos de Desenvolvimento (BRDE e BDMG), ou da Financiadora de Estudos e Projetos - FINEP. Nova pesquisa incluindo as contratações destas instituições poderia aumentar consideravelmente o tamanho da amostra proporcionando novas evidências. 


\section{Referências}

ALBANEZ, T. et al. Fatores institucionais e assimetria informacional: influência na estrutura de capital de empresas brasileiras. RAM. Revista de Administração Mackenzie, v. 13, n. 2, p. 76-105, 2012. DOI: 10.1590/S1678-69712012000200004

BACHILLER, J. V. Das Privatizações à Nova Estratégia de Desenvolvimento. A Trajetória do BNDES entre 1990 e 2010. Desenvolvimento em Questão, v. 14, n. 36, 2016. DOI: $10.21527 / 2237-6453.2016 .36 .5-30$

BARBOSA, G. DE C.; MEDEIROS, O. R. DE. Teste empírico da eficiência do mercado brasileiro na ocorrência de eventos favoráveis e desfavoráveis. Revista de Negócios, Blumenau, v. 12, n. 4, p. 44 - 54, outubro/dezembro 2007.

BARTHOLDY, J; OLSON, D.; PEARE, P. Conducting event studies on a small stock exchange. The European Journal of Finance, v. 13, n. 3, p. 227-252, 2007. DOI: $10.1080 / 13518470600880176$

BASTOS, D.; NAKAMURA, W. T. Determinantes da estrutura de capital das companhias abertas no Brasil, México e Chile no período 2001-2006. Revista Contabilidade \& Finanças-USP, v. 20, n. 50, 2009. DOI: 10.1590/S1519-70772009000200006

BROWN, S. J.; WARNER, J. B. Measuring security price performance. Journal of Financial Economics, v. 8, n. 3, p. 205-258, 1980. DOI: 10.1016/0304-405X(80)90002-1

Using daily stock returns: The case of event studies. Journal of Financial Economics, v. 14, n. 1, p. 3-31, 1985. DOI: 10.1016/0304-405X(85)90042-X

CAMARGOS, M. A. de; BARBOSA, F. V. Estudos de Evento: teoria e operacionalização. Caderno de Pesquisas em Administração, v. 10, n. 3, p. 01-20, 2003.

Eficiência informacional do mercado de capitais brasileiro pós-Plano Real: um estudo de eventos dos anúncios de fusões e aquisições. Revista de Administração-RAUSP, v. 41, n. 1, 2006.

Eficiência informacional do mercado de capitais brasileiro em anúncios de fusões e aquisições. Production, v. 25, n. 3, p. 571-584, 2015. DOI: 10.1590/0103-6513.0148T6

COPELAND, T. E.; WESTON J. F.; Shastri, K. Financial theory and corporate policy. Reading, Mass.: Addison-Wesley, 1983.

CORREA, C. A.; BASSO, L. F. C.; NAKAMURA, W. T. A estrutura de capital das maiores empresas brasileiras: análise empírica das teorias de pecking order e trade-off, usando panel data. Revista de Administração Mackenzie, v. 14, n. 4, 2013. DOI: 10.1590/S167869712013000400005

FAMA, E. F. Efficient capital markets: A review of theory and empirical work. The Journal of Finance, v. 25, n. 2, p. 383-417, 1970. DOI: 10.2307/2325486 
FAMÁ, R.; GRAVA, J. W. Teoria da estrutura de capital-as discussões persistem. Caderno de pesquisas em administração, v. 1, n. 11, p. 27-36, 2000.

FERRAZ, J. C. et al. O BNDES e o financiamento do desenvolvimento. Revista USP, n. 93, p. 69-80, 2012. DOI: 10.11606/issn.2316-9036.v0i93p69-80

GABRIEL, F. S.; RIBEIRO, R. B.; RIBEIRO, K. C. de S. Hipóteses de mercado eficiente: um estudo de eventos a partir da redução do IPI. Revista de Gestão, Finanças e Contabilidade, v. 3, n. 1, p. 36, 2013.

GAIO, L. E.; FREITAS ALVES, K. L. DE; PIMENTA JÚNIOR, T. O mercado acionário brasileiro do novo milênio: um teste de eficiência. BBR-Brazilian Business Review, v. 6, n. 3, 2009.

GRINBLATT, Mark; TITMAN, Sheridan. Mercados financeiros \& estratégia corporativa. Bookman Editora, 2016.

GRZEBIELUCKAS, C. et al. A estrutura de capital e a performance das firmas: uma análise empírica em companhias abertas no Brasil. REBRAE, v. 1, n. 1, p. 73-88, 2017. DOI:

10.7213/rebrae.v1i1.13316

HARRIS, M.; RAVIV, A. The theory of capital structure. The Journal of Finance, v. 46, n. 1, p. 297-355, 1991. DOI: 10.1111/j.1540-6261.1991.tb03753.x

JENSEN, M. C.; MECKLING, W. H. Theory of the firm: Managerial behavior, agency costs and ownership structure. Journal of financial economics, v. 3, n. 4, p. 305-360, 1976. DOI: $10.1016 / 0304-405 x(76) 90026-x$

KOTHARI, S. P.; WARNER, J. B. Econometrics of Event Studies, Handbook of Corporate Finance: Empirical Corporate Finance (Handbooks in Finance Series, Elsevier/NorthHolland), B. Espen Eckbo (ed.), 2005. DOI: 10.1016/B978-0-444-53265-7.50015-9

KRASNER, S. D. Power structures and regional development banks. International organization, v. 35, n. 2, p. 303-328, 1981. DOI: 10.1017/s0020818300032458

KUDLAWICZ, C.; SENFF, C. O.; BACH, T. M. O Desempenho Econômico e a Estrutura de Capital: Empresas Brasileiras a Luz da Fronteira de Eficiência. Revista de Globalización, Competitividad y Gobernabilidad, v. 9, n. 3, p. 40, 2015. DOI:

10.3232/GCG.2015.V9.N3.02

LOCATELLI, R. L.; NASSER, J.; MESQUITA, J. M. de C. Fatores determinantes da estrutura de capital no agronegócio: o caso das empresas brasileiras. Organizações Rurais \& Agroindustriais, v. 17, n. 1, 2015.

MACKINLAY, A. Craig. Event studies in economics and finance. Journal of economic literature, v. 35, n. 1, p. 13-39, 1997.

MALAQUIAS, R. F.; EID JUNIOR, W. Eficiência de mercado e desempenho de fundos multimercados. Revista Brasileira de Finanças, v. 11, n. 1, 2013. 
MODIGLIANI, F.; MILLER, M. H. The cost of capital, corporation finance and the theory of investment. The American economic review, v. 48, n. 3, p. 261-297, 1958.

MURCIA, F. C. de S.; MURCIA, F. Dal-Ri; BORBA, J. A. The Informational Content of Credit Ratings in Brazil: An Event Study. Revista Brasileira de Finanças, v. 11, n. 4, 2013.

MYERS, S. C. The capital structure puzzle. The Journal of Finance, v. 39, n. 3, p. 574-592, 1984. DOI: $10.3386 / w 1393$

Capital structure. Journal of Economic Perspectives, v. 15, n. 2, p. 81-102, 2001.

DOI: $10.1257 /$ jep.15.2.81

PEIXOTO, F. M. et al. Custo de capital, endividamento e sustentabilidade empresarial: um estudo no mercado de capitais brasileiro no período de 2009 a 2013. RACE-Revista de Administração, Contabilidade e Economia, v. 15, n. 1, p. 39-66, 2016. DOI: 10.18593/race.v15i1.6281

PRATES, D. M.; CINTRA, M. A. M.; FREITAS, M. C. P. O papel desempenhado pelo BNDES e diferentes iniciativas de expansão do financiamento de longo prazo no Brasil dos anos 90. Economia e Sociedade, v. 9, n. 2, p. 85-116, 2000.

PROCIANOY, J. L.; ANTUNES, M. A. Os efeitos das decisões de investimento das empresas sobre os preços de suas ações no mercado de capitais. In: ENANPAD, 25., p. 16383, 2001. Anais..., 2001.

RAJAN, R. G.; ZINGALES, L. What do we know about capital structure? Some evidence from international data. The Journal of Finance, v. 50, n. 5, p. 1421-1460, 1995. DOI: $10.3386 / \mathrm{w} 4875$

SALINGER, M. Value Event Studies. The Review of Economics and Statistics, v. 74, n. 4, p. 671-677, 1992. DOI: $10.2307 / 2109381$

SCHAPIRO, M. G. Novos parâmetros para a intervenção do Estado na economia: persistência e dinâmica da atuação do BNDES em uma economia baseada no conhecimento. [s.1.] Universidade de São Paulo, 2009. DOI: 10.11606/t.2.2009.tde19022010-152023

SILVA BRITO, G. A.; CORRAR, L. J.; BATISTELLA, F. D. Fatores determinantes da estrutura de capital das maiores empresas que atuam no Brasil. Revista Contabilidade \& Finanças-USP, v. 18, n. 43, 2007. DOI: 10.1590/s1519-70772007000100002

TARANTIN JUNIOR, Wilson; VALLE, Maurício R. do. Estrutura de capital: o papel das fontes de financiamento nas quais companhias abertas brasileiras se baseiam. Revista Contabilidade \& Finanças, v. 26, n. 69, p. 331-344, 2015. DOI: 10.1590/1808$057 \times 201512130$

TITMAN, Sheridan. The effect of capital structure on a firm's liquidation decision. Journal of financial economics, v. 13, n. 1, p. 137-151, 1984. DOI: 10.1016/0304-405x(84)90035-7 
; WESSELS, Roberto. The determinants of capital structure choice. The Journal of Finance, v. 43, n. 1, p. 1-19, 1988. DOI: 10.2307/2328319

TORRES FILHO, E. T.; COSTA, F. N. BNDES e o financiamento do desenvolvimento.

Economia e Sociedade, v. 21, n. 4, p. 975-1009, 2011. DOI: 10.1590/s0104-

06182012000400011

VALLE, M. R. do. Estrutura de capital de empresas brasileiras num ambiente de altas taxas de juros e na presença de fontes diferenciadas de financiamento. [s.1.] Universidade de São Paulo, 2008.

VALLE, M. R.; ALBANEZ, T. Juros altos, fontes de financiamento e estrutura de capital: o endividamento de empresas brasileiras no período 1997-2006. Revista de Contabilidade e Organizações, v. 6, n. 16, 2012. DOI: 10.11606/rco.v6i16.52667 